\title{
Feeding filaggrin: effects of L-histidine supplementation in atopic dermatitis
}

\author{
This article was published in the following Dove Press journal: \\ Clinical, Cosmetic and Investigational Dermatology \\ 5 October 2017 \\ Number of times this article has been viewed
}

\author{
Siao Pei Tan ${ }^{1,2}$ \\ Simon B Brown ${ }^{1,2}$ \\ Christopher EM Griffiths ${ }^{3}$ \\ Richard B Weller ${ }^{1,2}$ \\ Neil K Gibbs ${ }^{3,4}$ \\ IMRC Centre for Inflammation \\ Research, ${ }^{2}$ Department of \\ Dermatology, The University of \\ Edinburgh, Edinburgh, ${ }^{3}$ Dermatology \\ Centre, Division of Musculoskeletal \\ and Dermatological Sciences, \\ Salford Royal NHS Foundation Trust, \\ University of Manchester, Manchester, \\ ${ }^{4}$ Curapel, Life Sciences Hub Wales, \\ Cardiff, UK
}

Correspondence: Neil K Gibbs

clo Curapel, Life Sciences Hub Wales, 3

Assembly Square, Cardiff, CFIO 4PL, UK

Email neil.gibbs@manchester.ac.uk
Abstract: Atopic dermatitis (AD), also known as eczema, is one of the most common chronic skin conditions worldwide, affecting up to $16 \%$ of children and $10 \%$ of adults. It is incurable and has significant psychosocial and economic impacts on the affected individuals. AD etiology has been linked to deficiencies in the skin barrier protein, filaggrin. In mammalian skin, L-histidine is rapidly incorporated into filaggrin. Subsequent filaggrin proteolysis releases L-histidine as an important natural moisturizing factor (NMF). In vitro studies were conducted to investigate the influence of L-histidine on filaggrin processing and barrier function in human skin-equivalent models. Our further aim was to examine the effects of daily oral L-histidine supplementation on disease severity in adult $\mathrm{AD}$ patients. We conducted a randomized, double-blind, placebo-controlled, crossover, nutritional supplementation pilot study to explore the effects of oral L-histidine in adult AD patients $(n=24)$. In vitro studies demonstrated that L-histidine significantly increased both filaggrin formation and skin barrier function $(P<0.01$, respectively). Data from the clinical study indicated that once daily oral L-histidine significantly reduced $(P<0.003)$ AD disease severity by $34 \%$ (physician assessment using the SCORingAD tool) and 39\% (patient self-assessment using the Patient Oriented Eczema Measure tool) after 4 weeks of treatment. No improvement was noted with the placebo $(P>0.32)$. The clinical effect of oral L-histidine in AD was similar to that of mid-potency topical corticosteroids and combined with its safety profile suggests that it may be a safe, nonsteroidal approach suitable for long-term use in skin conditions that are associated with filaggrin deficits such as AD. Keywords: atopic dermatitis, eczema, filaggrin, L-histidine, amino acid, skin barrier, nutritional supplement

\section{Introduction}

Atopic dermatitis (AD) is a common, incurable, chronic inflammatory skin condition with a high prevalence in infants that causes considerable reduction in quality of life. ${ }^{1-4}$ Despite its prevalence and morbidity, few targeted therapies currently exist for AD. The mainstay of management being symptomatic relief based on the use of nonspecific anti-inflammatory topical steroids, calcineurin inhibitors, and systemic immunosuppressants such as azathioprine, cyclosporine, and prednisolone. ${ }^{4,5}$ These therapies are associated with adverse side effects, and there is a large unmet clinical need for the development of targeted AD therapies that are effective, economic, and safe for use, especially in younger children. ${ }^{5}$

A seminal report in $2006^{6}$ demonstrated that of any marker so far identified, lossof-function mutations in the gene for the epidermal barrier protein profilaggrin $(F L G)$ show the strongest association with AD. ${ }^{7,8}$ Profilaggrin, originally called "histidinerich protein" because of its very high $(\sim 10 \%)$ histidine content,${ }^{9}$ is a large $(>400 \mathrm{kDa})$ 
polypeptide synthesized in the epidermal granular layer. It accumulates in keratohyalin granules before dephosphorylation and processing, via lower weight intermediates, to $\sim 37$ kDa filaggrin monomers. ${ }^{10,11}$ Filaggrin aggregates cytokeratins 1 and 10 and other intermediate filaments in the granular layer of keratinocytes, "collapsing" them as part of the epidermal terminal differentiation process to form corneocytes and flattened squames that are critical for skin barrier function. ${ }^{12,13}$ Filaggrin is ultimately deiminated and cleaved by proteases, including kallikrein 5, caspase-14, elastase-2, matripase, and prostatin, into its component hygroscopic amino acids which are the major constituent of the "natural moisturizing factor" (NMF) which further contributes to barrier function through skin hydration and maintenance of stratum corneum acidity. ${ }^{14-16}$

Despite the genetic association between $F L G$ mutations and $\mathrm{AD}$ being the strongest of any marker, ${ }^{6}$ the majority of AD individuals are wild type for $F L G .{ }^{17}$ In these individuals, epigenetic effects linked to disease severity and inflammatory cytokine milieu reduce filaggrin processing and NMF levels, thereby impairing skin hydration and barrier integrity. ${ }^{18,19}$ Abnormal proteolytic processing of profilaggrin into functional filaggrin monomers due to protease-antiprotease imbalance may also play a role in the disease development in patients with wild-type $F L G .{ }^{20} \mathrm{~A}$ compromised skin barrier, whether due to FLG mutations or epigenetically compromised profilaggrin processing, results in xerosis, allergen ingress, and $\mathrm{AD}$ disease initiation and exacerbation. ${ }^{21}$

Much of the current research activity is aimed at further understanding the involvement of filaggrin in the etiology of $\mathrm{AD}$ and translating these insights into new therapeutic approaches for this chronic and disabling condition. ${ }^{7,21}$ Otsuka et a ${ }^{22}$ screened a 1120 compound library of bioactives and reported that JTC801, a four-aminoquinoline derivative, had the ability to increase $F L G$ transcription and translation in a human skin-equivalent model. A gene therapy approach has been used to successfully deliver a filaggrin monomer coding construct into the $F L G$-deficient (flaky tail) mouse model, thus restoring a normal skin barrier phenotype. ${ }^{23}$

In this paper, we suggest that a simpler, nutritional supplementation of L-histidine may have a beneficial potential in $\mathrm{AD}$.

L-histidine is a proteinogenic amino acid that is not synthesized by mammals. In human infants, it is considered "essential" due to low levels of histidine-synthesizing gut microflora and minimal carnosinase activity, which helps in releasing free L-histidine from carnosine. ${ }^{24}$ Our interest in the use of L-histidine in $\mathrm{AD}$ was stimulated by several observations. Firstly, in both infants and adults, a histidine-deficient diet results in an eczematous rash. ${ }^{25} \mathrm{In}$ rodents, ${ }^{3} \mathrm{H}$-histidine is rapidly (1-2 hours) incorporated into profilaggrin within keratohyalin granules after intraperitoneal or intradermal injection $^{14,26}$ and within 1-7 days is released as a free NMF amino acid in the upper stratum corneum. ${ }^{14}$ Furthermore, reduced stratum corneum levels of free NMF amino acids, including histidine and its acidifying metabolite urocanic acid (UCA), are associated with $\mathrm{AD}$ disease severity and FLG genotype. ${ }^{27,28}$

Given this evidence for the dependence of filaggrin processing and NMF formation on suitable levels of L-histidine, we hypothesized that $\mathrm{L}$-histidine would both enhance filaggrin processing in an in vitro, organotypic, human skin model and have beneficial effects as a nutritional supplement in subjects with atopic dermatitis.

\section{Methods}

\section{In vitro studies}

\section{Human keratinocyte culture condition}

Immortalized human $\mathrm{HaCaT}$ keratinocytes ${ }^{29}$ of passages 35-41 (gift from Dr J. Wood, University of Dundee; origin German Cancer Research Center (DKFZ), Heidelberg, Germany) were seeded in six-well cell culture plates (Corning Incorporated, Corning, NY, USA) in D-MEM/F-12 medium with GlutaMAX (Thermo Fisher Scientific, Waltham, MA, USA) supplemented with $10 \%$ fetal bovine serum and $1 \%$ penicillin/streptomycin. Monolayer cultures were typically confluent after 48-72 hours. From days 15-21, culture media were supplemented with additional $1-5 \mathrm{mM}$ of amino acids (L-lysine, L-histidine, or D-histidine; Sigma-Aldrich Co., St Louis, MO, USA). Cells were harvested and lysed with Laemmli buffer (Sigma-Aldrich Co.) on day 21.

\section{SDS-PAGE and Western-blotting}

Total cellular protein from $\mathrm{HaCaT}$ monolayers was resolved by $9 \%$ sodium dodecyl sulfate polyacrylamide gel electrophoresis (SDS-PAGE) and Western blotting using standard protocols. Primary antibodies against the following antigens were used: filaggrin (goat polyclonal antibody; Santa Cruz Biotechnology Inc., Dallas, TX, USA) and keratin 10 (rabbit monoclonal; Abcam, Cambridge, UK). Densitometric analysis was performed with the VersaDoc Imaging System (Bio-Rad Laboratories Inc., Hercules, CA, USA); all bands were background-corrected and normalized to keratin 10 .

\section{Organotypic skin-equivalent models and Lucifer Yellow penetration assay}

Adult human dermal fibroblasts (HDFs; Thermo Fisher Scientific) of passages 3-5 were mixed with rat tail Collagen 
I (Thermo Fisher Scientific) and left to set in six-well cell culture plates. After 20-30 minutes, HaCaTs of passages 35-41 were seeded on the apical aspect of the gels. Upon the $\mathrm{HaCaT}$ cells achieving confluency, the entire cultures (including the HDFs-containing collagen structures) were placed on plastic grids, creating an air-liquid interface with the apical aspect exposed to air. Skin-equivalent cultures were maintained for a total of 19 days and supplemented at days 13-19 with $5 \mathrm{mM}$ of either L-lysine, L-serine, or L-histidine (Sigma-Aldrich Co.). Samples were washed twice in PBS, fixed in formalin, embedded in paraffin, and sectioned. Standard protocols were used for hematoxylin and eosin staining. At day 19, the skin models exhibited keratin 10, involucrin, filaggrin, and loricrin (data not presented), particularly in the suprabasal layers, indicating epidermal-like differentiation.

For the penetration assay, $50 \mu \mathrm{L}$ of $5 \mathrm{mM}$ Lucifer Yellow $\mathrm{CH}$ dipotassium dye (Sigma-Aldrich Co.) was applied to the apical surface of each skin model in the center at 5-minute intervals for a total of 10 minutes, before being washed off with PBS, fixed in formalin, and embedded in paraffin. Dewaxed and hydrated $3 \mu \mathrm{m}$ transverse sections were visualized using fluorescence at 455-495/505-555 nm. Lucifer Yellow is a water-soluble fluorescent disulfonic acid anionic dye frequently used to study neuronal morphology. It has been used to assess the permeability barrier of mice and skin-equivalent models. ${ }^{30-33}$

Although our skin model did not show a well-established epidermal stratum corneum under hematoxylin and eosin staining, minimal dye penetration through the skin model was seen at 5 minutes, indicating a functional barrier, equating that of human epidermis. The dye penetration was not confluent throughout the entire sample. Therefore the average percentage dye penetration was calculated in three, nonoverlapping, consecutive microscope fields on each section (a total of five sections were scored from each skin model).

\section{Clinical nutritional supplementation pilot study}

Subjects

Adult ( $>18$ years of age) subjects with a diagnosis of $\mathrm{AD}$ according to "The U.K. Working Party's Diagnostic Criteria for Atopic Dermatitis" ${ }^{34}$ were recruited. Exclusion criteria were pregnancy or lactation, liver disease, exposure to natural or artificial ultraviolet radiation, immunosuppression due to disease or medication, or use of Chinese herbal medicine in the 3 months preceding the study. Subjects were permitted to continue the use of nonmedicinal emollients and intermittent rescue therapy of topical steroid creams, which were recorded in the case report forms at each visit.

\section{Study design}

This was a randomized, double-blind, placebo-controlled, crossover, nutritional supplement pilot study that examined the effects of L-histidine in adult subjects with AD. Subjects were randomized using Research Randomizer (www.randomizer.org) into either Group A or Group B. Initial AD disease severity was assessed by a trained dermatology research nurse using the validated SCORing Atopic Dermatitis (SCORAD) measure. ${ }^{35}$ The subjects were also trained to conduct the first of weekly self-assessments of their AD disease severity using the validated Patient Oriented Eczema Measure (POEM). ${ }^{35}$ After a 2-week wash-out period in which subjects were asked not to use any medicinal product for their AD, the same measures were repeated and patients were provided with identical sachets containing either $4 \mathrm{~g}$ L-histidine (Group A) or $4 \mathrm{~g}$ placebo (erythritol); Group B) which was taken once a day, dissolved in a morning fruit drink. Patients returned 4 and 8 weeks later and SCORAD was performed by a single, trained dermatology research nurse at each visit. Patients in Group A then crossed over to placebo and those in Group B took L-histidine for the next 8 weeks with SCORAD being performed by the trained dermatology research nurse at 4-weekly intervals and POEM questionnaires being completed at weekly intervals.

\section{Regulatory and ethics approval}

The UK Medicines and Healthcare Products Regulatory Agency confirmed that this nutritional supplementation pilot study on the effects of an amino acid was not classified as a "Clinical Trial of an Investigational Medicinal Product". Bolton Research Ethics Committee gave permission for the study (\#08/H1009/52) which was conducted in accordance with the Declaration of Helsinki 1964 and the EMEA Note for Guidance on Good Clinical Practice with written, informed consent obtained from all subjects.

\section{Statistics}

Analysis of variance (one- or two-way, with Dunnett's and Bonferroni post hoc tests performed, respectively) and simple linear regression analysis were used for the in-vitro studies. These were performed using GraphPad Prism version 4.00 for Windows (GraphPad software, San Diego, CA, USA). Data are shown as mean values \pm standard deviation.

Statistical analysis of the clinical pilot study was carried out by an independent statistical analyst (StatSol, Sereetz, 
Germany) using SPSS version 15. The data are shown as mean \pm standard errors and the significance of differences between means were expressed as a two-sided exact $P$-value of Wilcoxon rank-sum tests. In both the clinical and in vitro studies, $P$-values $<0.05$ were considered as significant.

\section{Results}

\section{L-histidine effects on profilaggrin processing and skin barrier function in vitro}

The addition of L-histidine to monolayer cultures of $\mathrm{HaCaT}$ keratinocytes $(\mathrm{N}=6)$ caused a decrease in a large $120 \mathrm{kDa}$ profilaggrin intermediate ${ }^{11}$ and a concomitant increase in $37 \mathrm{kDa}$ filaggrin monomers (Figure 1). This increase in the $37 \mathrm{kDa}: 120 \mathrm{kDa}$ ratio (mean 1.69, SD 0.46) was L-histidine dose $(0-5 \mathrm{mM})$ dependent $(P<0.01)$ but was not seen when keratinocytes were incubated with $5 \mathrm{mM}$ D-histidine (mean 0.68, SD 0.13) or L-lysine (mean 1.02, SD 0.37) (Figure 1).

To examine the effect of L-histidine on epidermal barrier function, organotypic skin-equivalent cultures (Figure 2A) were incubated with $5 \mathrm{mM}$ L-histidine (mean 3.60, SD 0.88) which led to a significant reduction in the penetration of Lucifer Yellow fluorescent dye $(\mathrm{N}=5, P<0.01)$ compared to the control (mean 7.67, SD 0.37). The same concentration of L-lysine (mean 8.78, SD 1.55) or L-serine (mean 7.64, SD 2.00) had no effect on the dye penetration (Figure 2B).

\section{Clinical nutritional supplementation pilot study \\ Subjects' demographics and $A D$ severity}

Twenty-four adults with AD were screened and randomized into two treatment groups. Patients in Group A received L-histidine in the treatment period 1 of 8 weeks, followed by placebo in treatment period 2, whilst patients in Group B received placebo followed by L-histidine (Figure 3A). Three patients (one in Group A and two in Group B) were lost to the study after the wash-out period (Figure 3B). The remaining patients entering the study had a mean (standard error of the mean [SEM]) age of 25.9 (1.6) years and 27.6 (1.6) years in Groups A and B, respectively; $55 \%$ of patients in Group A and $70 \%$ in Group B were females. The majority of patients
A

$120 \mathrm{kDa}$
profilaggrin
intermediate

Control

$5 \mathrm{mM}$ L-histidine
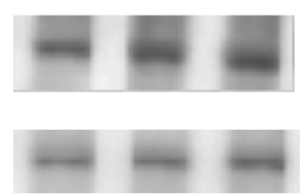

$37 \mathrm{kDa}$

filaggrin
Keratin 10 (loading control)
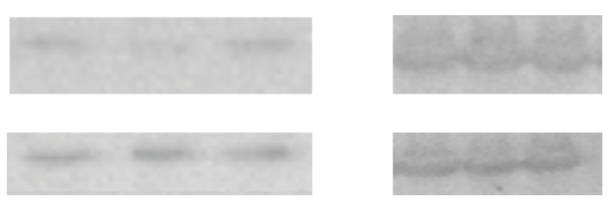

C

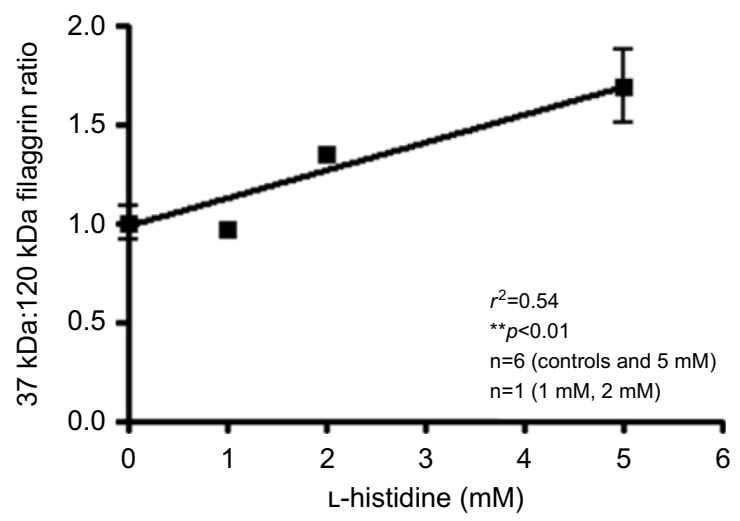

Figure I L-histidine increases filaggrin protein formation in confluent human (HaCaT) keratinocyte monolayers. (A) Representative Western blots showing a decrease in I20 $\mathrm{kDa}$ filaggrin and an increase in $37 \mathrm{kDa}$ filaggrin formation after treatment with L-histidine. (B) L-lysine and D-histidine had no significant effect on filaggrin protein expression while L-histidine increased the $37 \mathrm{kDa}$ to $120 \mathrm{kDa}$ filaggrin ratio $(P<0.0 \mathrm{I})$, as compared to controls. (C) L-histidine increased the $37 \mathrm{kDa}$ : $20 \mathrm{kDa}$ filaggrin ratio in a dosedependent manner $\left(R^{2}=0.54, P<0.0 \mathrm{I}\right)$. Error bars represent mean $\pm \mathrm{SD}$, where $\mathrm{n}=6$. All bands were standardized to housekeeping protein keratin I0 loading control. ** $\mathrm{p}<0.0 \mathrm{I}$. Abbreviations: OD, optical density; WB, Western blot. 
A

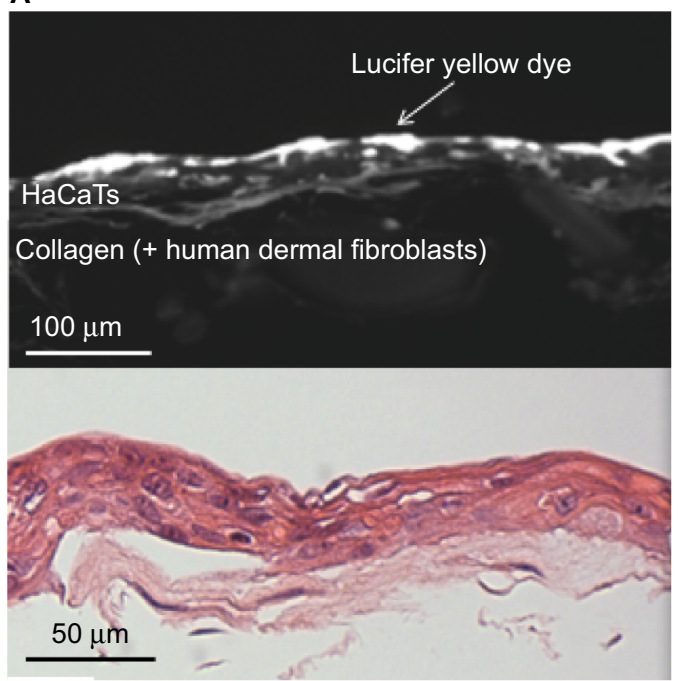

B

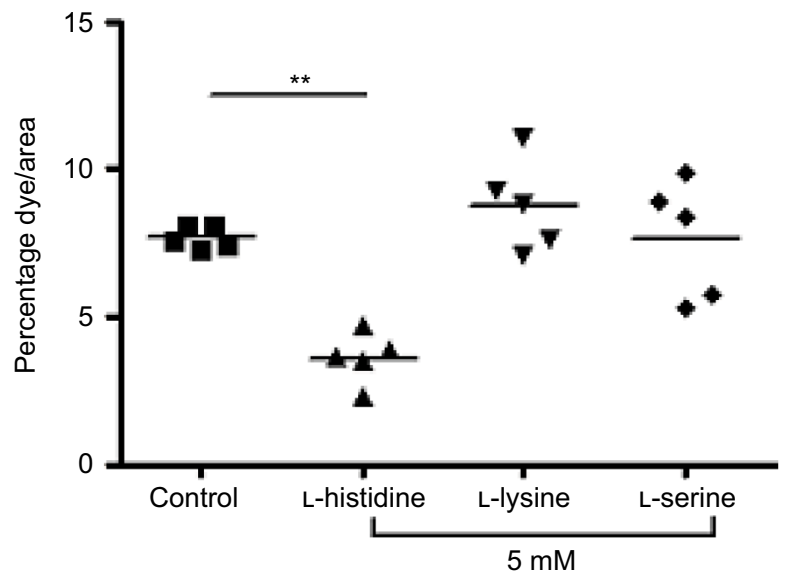

Figure 2 L-histidine enhances the barrier function of organotypic skin model as indicated by penetration of Lucifer Yellow fluorescent dye. (A) A representative image of organotypic skin model with Lucifer Yellow dye seen under fluorescence and H\&E staining. (B) Skin models grown in $5 \mathrm{mM} L$-histidine had reduced dye penetration ( $\mathrm{N}=5$; $P<0.0$ I), whereas L-lysine and L-serine had no effect on barrier function.

Note: $* * 00.01, \mathrm{n}=5$.

Abbreviation: H\&E, hematoxylin and eosin.

A

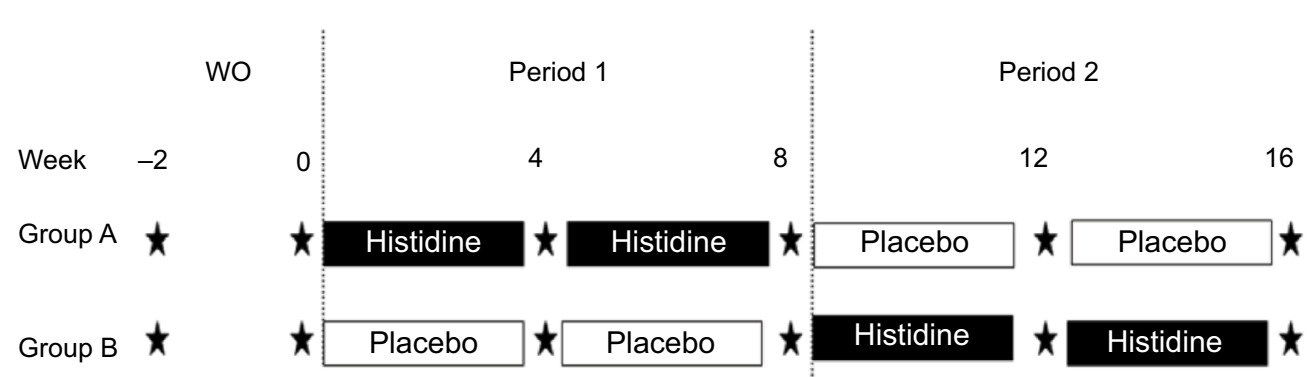

Clinic visits when SCORAD was recorded

B

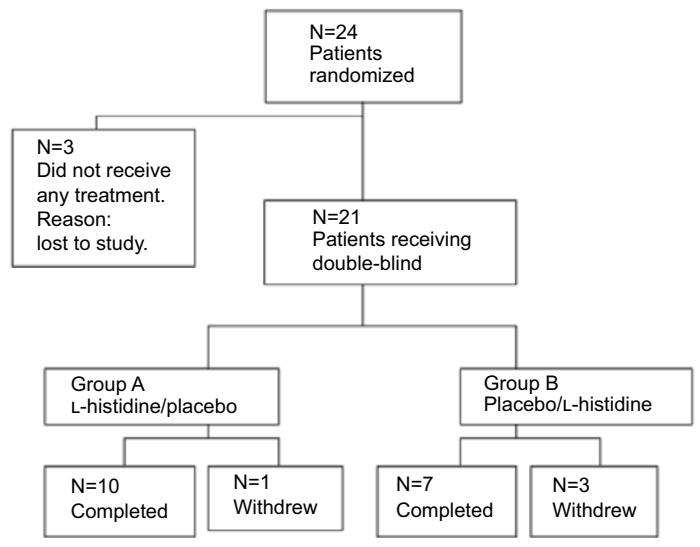

C

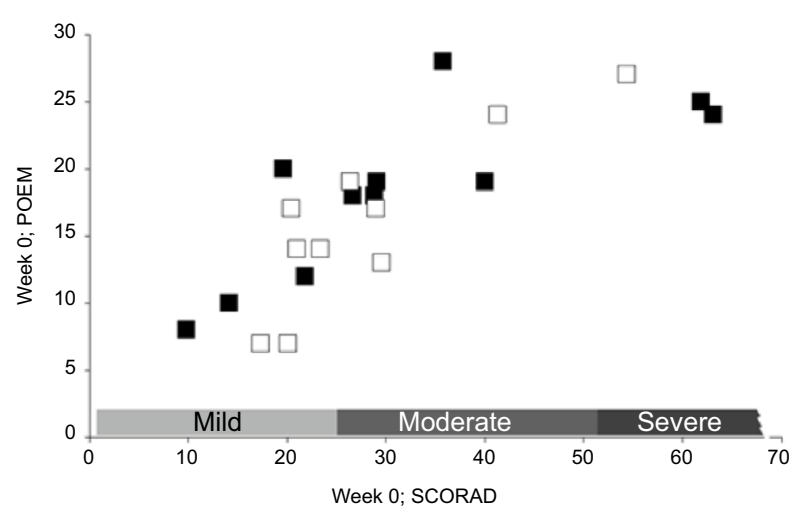

Figure 3 Clinical study protocol and patient details. (A) Schema showing the study protocol, (B) patients completed POEM questionnaires weekly, and (C) disposition of patients. There was a good correlation between SCORAD and POEM scores $\left(R^{2}=0.62\right)$ in study patients in Group A ( $\left.\square\right)(\mathrm{n}=1 \mathrm{I})$ and Group B ( $\left.\square\right)(\mathrm{n}=10)$ at week 0 . There was no difference in mean SCORAD or POEM scores between the two groups $(P=0.86)$.

Abbreviations: POEM, Patient Oriented Eczema Measure; SCORAD, SCORing Atopic Dermatitis; WO, washout period. 
were Caucasians with one patient of Asian and one of Asian/ Caucasian race in Groups A and B, respectively.

Both clinician-scored SCORAD and patient-scored POEM were used as validated measures of AD disease severity. ${ }^{35}$ At week 0 , there was no significant difference in the mean (SEM) SCORAD (31.9 [5.3] and 28.3 [3.6]) and POEM (18.4 [1.9] and 16.7 [6.2]) scores between Groups $A(\mathrm{~N}=11)$ and $\mathrm{B}(\mathrm{N}=10)$, respectively. Across all patients, there was a strong correlation between SCORAD and POEM scores at week $0\left(R^{2}=0.62\right)$ (Figure $\left.3 \mathrm{C}\right)$.

\section{Effects of L-histidine nutritional supplementation on $A D$ severity}

Following L-histidine (period 1) supplementation, there was a significant reduction from week 0 scores in $\operatorname{SCORAD}(34 \%$, $P=0.0029$ and $32 \%, P=0.0029$; Figure $4 \mathrm{~A})$ and $P O E M(39 \%$, $P=0.0020$ and $39 \%, P=0.0010$; Figure 4B) scores in Group A at weeks 4 and 8, respectively. No significant reduction in disease severity was seen in Group B which received placebo during period 1 at weeks 4 or 8 (SCORAD: $-16 \%$, $P=0.3223$ and $6 \%, P=0.5391$; POEM: $2 \%, P=0.8438$ and $16 \%, P=0.2695$ ), respectively (Figure $4 \mathrm{~A}$ and $\mathrm{B}$ ). In period 2 , there was evidence that subjects in Group B, who crossed over from placebo to L-histidine, showed an improvement in their AD disease severity, although a carry-over effect of L-histidine in Group A over this period prevented meaningful analysis of study after the crossover.

\section{Adverse events}

Potential adverse events were monitored and recorded over the course of the study. There were no adverse events directly associated with administration of either L-histidine or the placebo.

\section{Discussion}

Current AD therapy is based on the palliative use of emollients and anti-inflammatory agents such as topical corticosteroids or calcineurin inhibitors, with treatment of

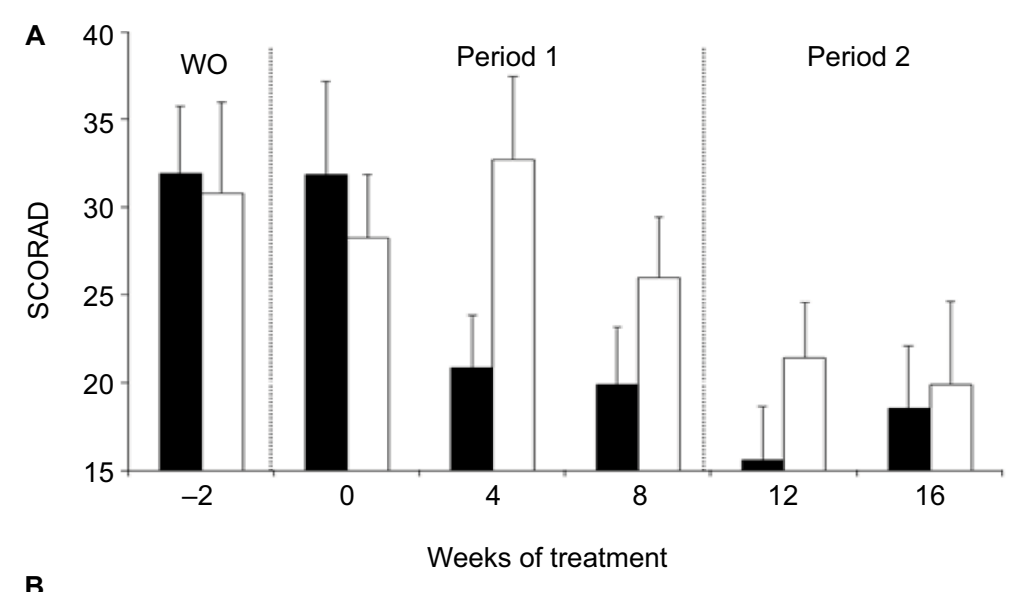

B

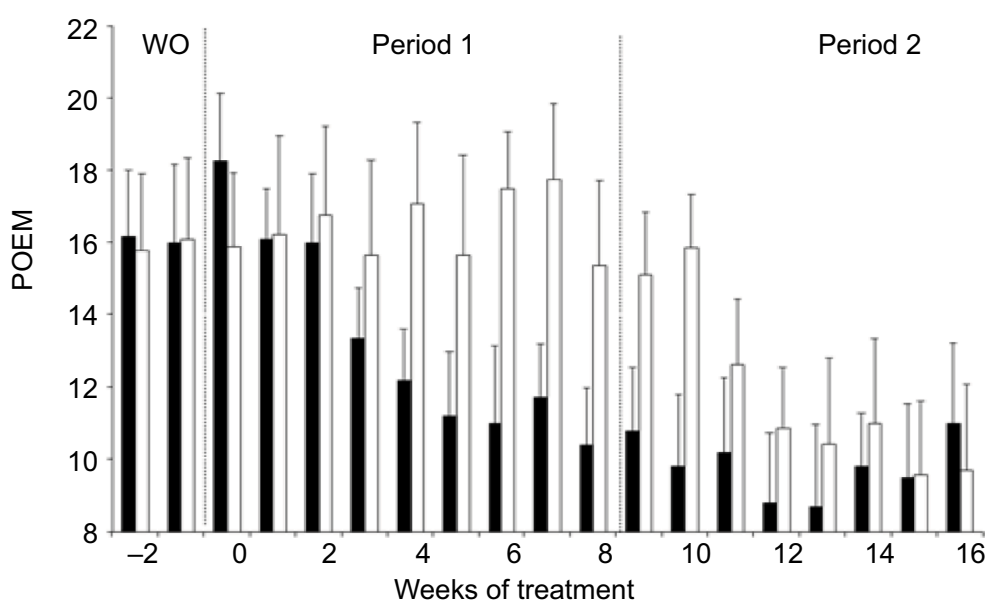

Figure 4 Effects of L-histidine nutritional supplementation on $A D$ disease severity. (A) SCORAD and (B) POEM scores (mean \pm SEM) were significantly reduced in Group $A$ ( $\square$ ) patients at weeks 4 and 8 (SCORAD, $P=0.0029$ and $P=0.0029$; POEM, $P=0.0020$ and $P=0.0010$, respectively) in period I, whilst the placebo had no effect in Group $B$ ( $\square$ ) patients in period I (SCORAD, $P=0.3223$ and $P=0.539$ I; $P O E M, P=0.8438$ and $P=0.2695$, respectively). There is a clear "carry-over"effect of $L$-histidine in $G$ roup $A$ between weeks 8 and I2, which precludes meaningful statistical analysis within the study period 2.

Abbreviations: AD, atopic dermatitis; POEM, Patient Oriented Eczema Measure; SCORAD, SCORing Atopic Dermatitis; SEM, standard error of the mean; WO, washout period. 
superinfection, particularly due to Staphylococcus aureus and Herpes simplex, when it arises. If topical management is unsuccessful, systemic treatment based on potent drugs such as corticosteroids, methotrexate, azathioprine, ciclosporin A, or mycophenolate mofetil is needed. Well-known effects of long-term use of systemic corticosteroids include osteoporosis, cataracts, hypertension, and hyperglycemia. Immunosuppressants such as cyclosporin A and azathioprine also have serious potential side effects including hematological abnormalities, predisposition to life-threatening infections, liver and renal failure, therefore requiring intensive monitoring by the supervising doctor. ${ }^{5-36}$ Given the high prevalence of $\mathrm{AD}$ (up to $16 \%$ of children ${ }^{1}$ and $10 \%$ of adults $^{2}$ worldwide), these adverse effects impose a considerable burden on the individual patient and a high financial cost for health care systems and society. Clinical trials are currently being undertaken with biologic agents acting on elements of the immune system, but if effective, these will be expensive and are likely to be limited to the most severe and treatment-resistant AD. Concerns over the safety of this class of agents continue, particularly over increased infection risks and malignancy. ${ }^{5,36}$ A large unmet clinical need therefore remains for safe, convenient, targeted nonsteroidal interventions suitable for long-term use in the management of $\mathrm{AD}$, particularly in children.

$\mathrm{HaCaT}$ monolayers grown in L-histidine-enriched media were associated with significantly increased expression of the $37 \mathrm{kDa}$ filaggrin monomers relative to the $120 \mathrm{kDa}$ filaggrin intermediate. No increase in the expression of the filaggrin monomers was seen with media enriched with L-lysine and D-histidine, the structurally similar but biologically inactive isomers of L-histidine. The mechanism by which L-histidine enhanced filaggrin processing in an enantiomer-specific manner is unclear, although L-histidine is a common participant in enzymatic reactions ${ }^{37}$ owing to the amphotericity of its imidazole side chain.

The $37 \mathrm{kDa}$ filaggrin monomers are intermediate products of filaggrin proteolysis which are further degraded into smaller filaggrin peptide fragments by proteases including calpain-1 and caspase-14 and finally into free amino acids by bleomycin hydrolase..$^{38}$ Increased formation of the 37 $\mathrm{kDa}$ filaggrin monomers by L-histidine would be expected to improve keratin aggregation and lead to increased levels of free amino acid NMF components. L-histidine is hygroscopic, and this ability to capture and retain water makes it an important component of the NMF. ${ }^{14}$ The ability of L-histidine to increase filaggrin processing with consequent enhancement of skin barrier function is supported by our finding that skin equivalents grown in L-histidine-enriched media were more resistant to penetration by Lucifer Yellow fluorescent dye. The observed effect may be due to either improvement in keratin aggregation or increased level of NMF in the skin models due to increased substrate (i.e., more filaggrin monomers) availability for proteolytic degradation, or both. In individuals with wild-type $F L G$ or heterozygous loss-offunction $F L G$ mutations, L-histidine may improve the disease symptoms by enhancing filaggrin formation and supplement NMF production, whilst in patients with homozygous $F L G$ mutations, L-histidine may increase the amount of NMF in the skin. In all cases, enhancement of filaggrin formation and/or supplementing NMF would be expected to enhance skin barrier function and reduce the disease burden of AD.

The use of primary human keratinocytes instead of $\mathrm{HaCaT}$ cells for the barrier function assay may be argued to be more "physiological". However, our in-house skin-equivalent model is an adaptation of the technique demonstrated by Schoop et al, ${ }^{39}$ who have shown that HaCaT cells, cultured at an air-liquid interface on various matrixes serving as dermal equivalents, can be used to generate highly differentiated organotypic skin-equivalent structures in vitro. Unlike primary keratinocytes which are normally derived from human foreskin samples, the HaCaT cell line is of standardized quality as it is independent of donor variations.

The clinical nutritional supplementation pilot study suggests that oral L-histidine, administered once daily over a period of 4 weeks, is associated with improvement in the clinical signs and symptoms of adult AD patients. In both clinician-scored (SCORAD) and patient-scored (POEM) measures of disease severity, there was an $\sim 40 \%$ decrease in $\mathrm{AD}$ activity over 4 weeks of treatment which is similar to that reported from using mid-potency (Group III) topical corticosteroids. ${ }^{40}$ The beneficial effects seen in Group A patients persisted for several weeks following crossover from L-histidine to the placebo suggesting a prolonged benefit of the L-histidine treatment. Importantly, no adverse events were reported that were attributable to L-histidine supplementation.

We have demonstrated, in vitro, a L-histidine-concentration dependent increase in $37 \mathrm{kDa}$ filaggrin monomer formation and L-histidine-associated improvement in the barrier function of a skin-equivalent model. This observation correlates with evidence from the clinical nutritional pilot study that oral L-histidine may have therapeutic benefits in AD. Although there are limitations relating to the sample size of our pilot study, if consistent, the results suggest that once-a- day oral L-histidine has similar effects in $\mathrm{AD}$ to those reported for mid-potency (Group III) topical corticosteroids. ${ }^{40}$ 
These observations, when combined with its established safety profile, suggest that L-histidine nutritional supplementation may be a safe, convenient, nonsteroidal intervention suitable for long-term use in the management of $\mathrm{AD}$, particularly in children.

\section{Acknowledgments}

This study was supported by grants from the Scottish Overseas Research Student Award, University of Edinburgh College of Medicine and Veterinary Medicine PhD Scholarship, and the University of Manchester. CEMG is a Senior Investigator in National Institute for Health Research. We thank the Manchester Dermatology Centre research nurses for managing the clinical part of this project and also thank all patient volunteers for participating in this study.

\section{Author contributions}

All authors contributed toward data analysis, drafting and critically revising the paper, gave final approval of the version to be published, and agree to be accountable for all aspects of the work.

\section{Disclosure}

CEMG reports grants from Zymogenetics, Stiefel, and Regeneron, grants and personal fees from Novartis and Pfizer. NKG is a founding director of Curapel, a University of Manchester spin-out company owning patents in this field. The other authors report no conflicts of interest in this work.

\section{References}

1. Williams H, Robertson C, Stewart A, et al. Worldwide variations in the prevalence of symptoms of atopic eczema in the International Study of Asthma and Allergies in Childhood. J Allergy Clin Immunol. 1999;103:125-138.

2. Rönmark EP, Ekerljung L, Lötvall J, et al. Eczema among adults: prevalence, risk factors and relation to airway diseases. Results from a large-scale population survey in Sweden. Br J Dermatol. 2012;166:1301-1308.

3. Watson W, Kapur S. Atopic dermatitis. Allergy Asthma Clin Immunol. 2011;7 (Suppl 1):S4.

4. Weidinger S, Novak N. Atopic dermatitis. Lancet. 2016;387:1109-1122.

5. Katoh N. Future perspectives in the treatment of atopic dermatitis. J Dermatol. 2009;36:367-376.

6. Palmer CNA, Irvine AD, Terron-Kwiatkowski A, et al. Common loss-offunction variants of the epidermal barrier protein filaggrin are a major predisposing factor for atopic dermatitis. Nat Genet. 2006;38:441-446.

7. Brown SJ, McLean WHI. Eczema genetics: current state of knowledge and future goals. J Invest Dermatol. 129:543-552.

8. Irvine AD, McLean WHI, Leung DYM. Filaggrin mutations associated with skin and allergic diseases. N Engl J Med. 2011;365:1315-1327.

9. Voorhees JJ, Chakrabarti SG, Bernstein IA. The metabolism of "histidine-rich" protein in normal and psoriatic keratinization. J Invest Dermatol. 1968;51:344-354.

10. Brown SJ, McLean WHI. One remarkable molecule: filaggrin. J Invest Dermatol. 2012;132:751-762.
11. Robertson ED, Weir L, Romanowska M, Leigh IM, Panteleyev AA. ARNT controls the expression of epidermal differentiation genes through HDAC- and EGFR-dependent pathways. J Cell Sci. 2012;125:3320-3332.

12. Lynley AM, Dale BA. The characterization of human epidermal filaggrin. A histidine-rich, keratin filament-aggregating protein. Biochim Biophys Acta. 1983;744:28-35.

13. Gruber R, Elias PM, Crumrine D, et al. Filaggrin genotype in ichthyosis vulgaris predicts abnormalities in epidermal structure and function. $\mathrm{Am}$ J Pathol. 2011;178:2252-2263.

14. Scott IR, Harding CR, Barrett JG. Histidine-rich protein of the keratohyalin granules. Source of the free amino acids, urocanic acid and pyrrolidone carboxylic acid in the stratum corneum. Biochim Biophys Acta. 1982;719:110-117.

15. Hoste E, Kemperman P, Devos M, et al. Caspase-14 is required for filaggrin degradation to natural moisturizing factors in the skin. J Invest Dermatol. 2011;131:2233-2341.

16. McAleer MA, Irvine AD. The multifunctional role of filaggrin in allergic skin disease. J Allergy Clin Immunol. 2013;131:280-291.

17. Brown SJ, Relton CL, Liao H, et al. Filaggrin null mutations and childhood atopic eczema: a population-based case-control study. J Allergy Clin Immunol. 2008;121:940-946.e3.

18. Kezic S, O'Regan GM, Yau N, et al. Levels of filaggrin degradation products are influenced by both filaggrin genotype and atopic dermatitis severity. Allergy. 2011;66:934-940.

19. Howell MD, Kim BE, Gao P, et al. Cytokine modulation of atopic dermatitis filaggrin skin expression. J Allergy Clin Immunol. 2009;124:R7-R12.

20. Tan SP, Abdul-Ghaffar S, Weller RB, Brown SB. Protease-antiprotease imbalance may be linked to potential defects in profilaggrin proteolysis in atopic dermatitis. Br J Dermatol. 2012;166:1137-1140.

21. Cabanillas B, Novak N. Atopic dermatitis and filaggrin. Curr Opin Immunol. 2016;42:1-8.

22. Otsuka A, Doi H, Egawa G, et al. Possible new therapeutic strategy to regulate atopic dermatitis through upregulating filaggrin expression. J Allergy Clin Immunol. 2014;133:139-146.e1-10.

23. Stout TE, McFarland T, Mitchell JC, Appukuttan B, Stout JT. Recombinant filaggrin is internalized and processed to correct filaggrin deficiency. J Invest Dermatol. 2014;134:423-429.

24. Bando K, Shimotsuji T, Toyoshima H, Hayashi C, Miyai K. Fluorometric assay of human serum carnosinase activity in normal children, adults and patients with myopathy. Ann Clin Biochem. 1984;21(Pt 6): 510-514.

25. Kopple JD, Swendseid ME. Evidence that histidine is an essential amino acid in normal and chronically uremic man. J Clin Invest. 1975;55: 881-891.

26. Fukuyama K, Nakamura T, Benstein IA. Differentially localized incorporation of amino acids in relation to epidermal keratinization in the newborn rat. Anat Rec. 1965;152:525-535.

27. Tanaka M, Okada M, Zhen YX, et al. Decreased hydration state of the stratum corneum and reduced amino acid content of the skin surface in patients with seasonal allergic rhinitis. $\mathrm{Br} J$ Dermatol. 1998;139:618-621.

28. Kezic S, O'Regan GM, Yau N, et al. Levels of filaggrin degradation products are influenced by both filaggrin genotype and atopic dermatitis severity. Allergy. 2011;66:934-940.

29. Boukamp P, Petrussevska R, Breitkreutz D, Hornung J, Markham A, Fusenig N. Normal keratinization in a spontaneously immortalized aneuploid human keratinocyte cell line. J Cell Biol. 1988;106:761-771.

30. McMahon A, Butovich IA, Kedzierski W. Epidermal expression of an Elov14 transgene rescues neonatal lethality of homozygous Stargardt disease-3 mice. J Lipid Res. 2011;52:1128-1138.

31. Herrmann T, Gröne H-J, Langbein L, et al. Disturbed epidermal structure in mice with temporally controlled Fatp4 deficiency. J Invest Dermatol. 2005; $125: 1228-1235$

32. Jennemann R, Sandhoff R, Langbein L, et al. Integrity and barrier function of the epidermis critically depend on glucosylceramide synthesis. J Biol Chem. 2007;282:3083-3094. 
33. Epp N, Fürstenberger G, Müller K, et al. 12R-lipoxygenase deficiency disrupts epidermal barrier function. $J$ Cell Biol. 2007;177: 173-182.

34. Williams HC, Burney PG, Pembroke AC, Hay RJ. The U.K. Working Party's Diagnostic Criteria for Atopic Dermatitis. III. Independent hospital validation. Br J Dermatol. 1994;131:406-416.

35. Schmitt J, Langan S, Williams HC. What are the best outcome measurements for atopic eczema? A systematic review. JAllergy Clin Immunol. 2007;120:1389-1398.

36. Walling HW, Swick BL. Update on the management of chronic eczema: new approaches and emerging treatment options. Clin Cosmet Investig Dermatol. 2010;3:99.
37. Meth-Cohn O, Barton D, Nakanishi K. Comprehensive Natural Products Chemistry. London: Elsevier Science Ltd;1999:459.

38. Kamata Y, Taniguchi A, Yamamoto M, et al. Neutral cysteine protease bleomycin hydrolase is essential for the breakdown of deiminated filaggrin into amino acids. $J$ Biol Chem. 2009;284:12829-12836.

39. Schoop VM, Mirancea N, Fusenig NE. Epidermal organization and differentiation of $\mathrm{HaCaT}$ keratinocytes in organotypic coculture with human dermal fibroblasts. J Invest Dermatol. 1999;112:343-353.

40. Kirkup ME, Birchall NM, Weinberg EG, Helm K, Kennedy CTC. Acute and maintenance treatment of atopic dermatitis in children two comparative studies with fluticasone propionate $(0.05 \%)$ cream. J Dermatolog Treat. 2003;14:141-148.
Clinical, Cosmetic and Investigational Dermatology

\section{Publish your work in this journal}

Clinical, Cosmetic and Investigational Dermatology is an international, peer-reviewed, open access, online journal that focuses on the latest clinical and experimental research in all aspects of skin disease and cosmetic interventions. This journal is included on PubMed. The manuscript management system is completely online

\section{Dovepress}

and includes a very quick and fair peer-review system, which is all easy to use. Visit http://www.dovepress.com/testimonials.php to read real quotes from published authors

Submit your manuscript here: https://www.dovepress.com/clinical-cosmetic-and-investigational-dermatology-journal 